\title{
Aspectos históricos de la clinoterapia.
}

\author{
Historical aspects of clinotherapy.
}

Marco Macavilca-Cruz ${ }^{1, a}$; Santiago Stucchi-Portocarrero ${ }^{1,2, a}$

\section{RESUMEN}

La clinoterapia o "reposo en la cama" fue introducida como tratamiento para dolencias mentales en 1852, con el propósito de inhibir la "conciencia de movimiento" y la excitación periférica y, así, posibilitar un "reposo psíquico". En un primer momento la clinoterapia, junto con otras medidas como el open door y el non restraint, significó una alternativa a las celdas de reclusión, las camisas de fuerza y otros métodos restrictivos. Sin embargo, a la postre se vio reducida al simple aislamiento en una habitación, muchas veces en condiciones muy poco apropiadas.

PALABRAS CLAVE: Historia de la medicina, reposo en cama, psiquiatría, clinoterapia.

\section{SUMMARY}

Clinotherapy or "bed rest" was first introduced as a treatment for mental illness in 1852, with the aim of inhibit the "consciousness of movement" and peripheral excitation, in order to make it possible a "psychological rest". At the beginning, clinotherapy, along with open door and non-restraint principles, meant an alternative to seclusion rooms, straitjackets and other restrictive practices. However, over time it became a simple seclusion in a room, many times in inappropriate conditions.

KEYWORDS: History of medicine, bed rest, psychiatry, clinotherapy.

\section{INTRODUCCIÓN}

Sabemos que desde sus albores, la medicina se ha dedicado a atender a los enfermos con diversos tratamientos, entre los cuales el reposo en cama fue imprescindible. Es menester de este artículo referir la evolución histórica de aquella medida en los "locos", "insanos" o "enfermos del alma": cómo en un primer momento se implementó con gran entusiasmo científico y como una alternativa más humana frente a otros métodos restrictivos; cómo luego se fueron implementando medidas coercitivas para obligar a los enfermos a permanecer en sus camas, y cómo finalmente el descanso en cama se vio reducido conceptualmente al simple aislamiento en una habitación, muchas veces en condiciones no apropiadas.
El término clinoterapia proviene de la raíz griega $\kappa \lambda \imath \omega \omega$ (klino/klinein): $\operatorname{acostar}(1)$, reclinarse (2). Dicha raíz se evidencia en términos como: in-clinarse, proclive, de-clinar, y es más directa la derivación en el vocablo "clínica" (lugar donde uno se acuesta) (1). En esta línea, Brandam, en su tesis de 1901, definió a la clinoterapia como el "reposo del cuerpo" o el "reposo en el lecho", lo que se asemeja al concepto de "reposo absoluto" (3). El terminó trascendió el ámbito médico; por ejemplo, el poeta colombiano León de Greiff lo usó en sus versos, lo que hizo que fuera incluida por expertos en el glosario de referencias léxicas sobre su obra, donde definieron clinoterapia como: "Terapia de inclinarse, o de ser sumiso ante los otros. Hacerles venia" (4).

\footnotetext{
Facultad de Medicina, Universidad Peruana Cayetano Heredia. Lima, Perú

2 Hospital Víctor Larco Herrera.Lima, Perú

a Médico psiquiatra.
} 
El británico John Conolly fue el precursor del tratamiento en el lecho, al indicarlo en diversas formas de locura. Siguiendo a Pinel y a sus compatriotas Charlesworth y Hill, abandonó todo procedimiento de contención mecánica en los manicomios y se constituyó, desde 1839, como el impulsor del principio del non restraint $(3,5,6)$. Como parte del mismo, Connolly también introdujo el concepto de reclusión (seclusion en inglés), creado paradójicamente con la intención de liberar a los locos de la sujeción mecánica y otras medidas coercitivas, considerándolo como la forma más humana de tratar a quienes presentaban riesgo para su integridad y la de otros, antes que como una medida restrictiva (7).

Posteriormente, alrededor de 1852, fue el belga Joseph Guislain quien impulsó el uso de la clinoterapia como opción terapéutica, y contribuyó a su divulgación científica. Escritos suyos mencionaron lo siguiente (citado por Brandam): "Casi todos nuestros melancólicos están acostados Yo prescribo el reposo del cuerpo. La cama será durante todo el periodo del mal, uno de los grandes recursos del tratamiento. Desde luego el paciente será acostado de noche y durante una gran parte del día, se levantará de tiempo en tiempo, quedando sentado durante una hora ó dos, para acostarse de nuevo". No queda duda de su entusiasmo frente a la efectividad de este tratamiento: "No se imagina como el decúbito prolongado facilita en los alienados el retorno de la calma. Así mis cuidados tienden sin cesar á disminuir el tumulto y la agitación, á recomendar el reposo, a favorecer el sueño. (...) Yo lo digo con íntima convicción: ningún medio me ha dado resultado más satisfactorio en el tratamiento de la melancolía..." (3).

Fue tal el interés por este tratamiento que pronto se empezó a aplicar en otros países. En Alemania fue utilizado por Heinrich Laehr de Schweizerhof (1854) y Clemens Neisser (1890); este último la denominó Bettbehandlung (2,3). En Francia, la aplicó J. P. Falret padre (1864). Y en Rusia, fue impulsada por Timoféiew (1892) y Govséiew (1895) (3).

Por otro lado, en los Estados Unidos, Weir Mitchell (1875) extendió el uso de la clinoterapia a estados que podrían denominarse "neuróticos", tales como la histeria y la neurastenia (3), siendo esta última una condición que afectaba a "mujeres nerviosas, quienes como regla son delgadas y escasas de sangre" (8). Mitchell prescribió la cura en el lecho (rest cure) casi exclusivamente a mujeres, e incluyó tres elementos principales: aislamiento, reposo y alimentación, con electroterapia y masajes adicionales para contrarrestar la atrofia muscular de las largas sesiones de reposo, que podían durar hasta 24 horas continuas (8). En la misma línea, en España encontramos referencias al uso del reposo en el lecho junto a la sugestión, el aislamiento y la reeducación para el tratamiento de los "trastornos nerviosos", con un carácter claramente psicoterapéutico (9).

Mientras tanto en Argentina, Domingo Cabred, Director del Hospicio de las Mercedes, aplicó desde 1897 y a gran escala el tratamiento en el lecho, con el cual "las viejas celdas quedan desiertas" $(2,3)$. Dicho nosocomio llegó a contar con una Sección o Sala de Clinoterapia, que tuvo vigencia durante la primera mitad del siglo XX, como se pudo verificar en historias clínicas y algunas descripciones en medios escritos (10-12). Pocos años después, en Brasil, Juliano Moreira, al asumir la dirección del Hospicio Pedro II en 1903, inició una serie de reformas, fundó laboratorios de bacteriología y virología, desestimó el uso de chalecos, camisas de fuerza y rejas, y en su lugar promovió la clinoterapia y la filosofía del open door (13).

En el Perú, el Asilo Colonia de la Magdalena (actual Hospital Víctor Larco Herrera), inaugurado en 1918, contó tempranamente con pabellones de clinoterapia (ver la figura 1) (14). En 1937, Baltazar Caravedo consideró al reposo en el lecho como uno de los tres pilares de la evolución de la atención de los alienados, junto al open-door y el non restraint, en contraste con los "métodos de contención mecánica", que "excitaban más a los alienados", y "el sistema de clausura permanente", que "los deprimía y los humillaba". Además mencionó que para su aplicación en otras latitudes se diseñaban secciones especiales de observación y vigilancia continua, a cargo de enfermeros, en reemplazo del personal empírico (6). Es de notar que, inclusive en 1948, la Dirección del Hospital Víctor Larco Herrera solicitaba la construcción de "Una Clinoterapia para el mismo Servicio con capacidad de 50 camas" (15).

Algunos autores han antagonizado la clinoterapia a la terapia por el trabajo. Por ejemplo, para Turnes "el más rudo golpe a la terapia por el trabajo se lo asestó la última novedad terapéutica del siglo, la clinoterapia, consistente en meter al enfermo en la cama" (16). Lo cierto es que con el transcurrir del siglo XX, fue finalmente la terapia por el trabajo la que mantuvo vigencia, a la par que se fue desvaneciendo el uso terapéutico de la clinoterapia. Así, en el Asilo Colonia 


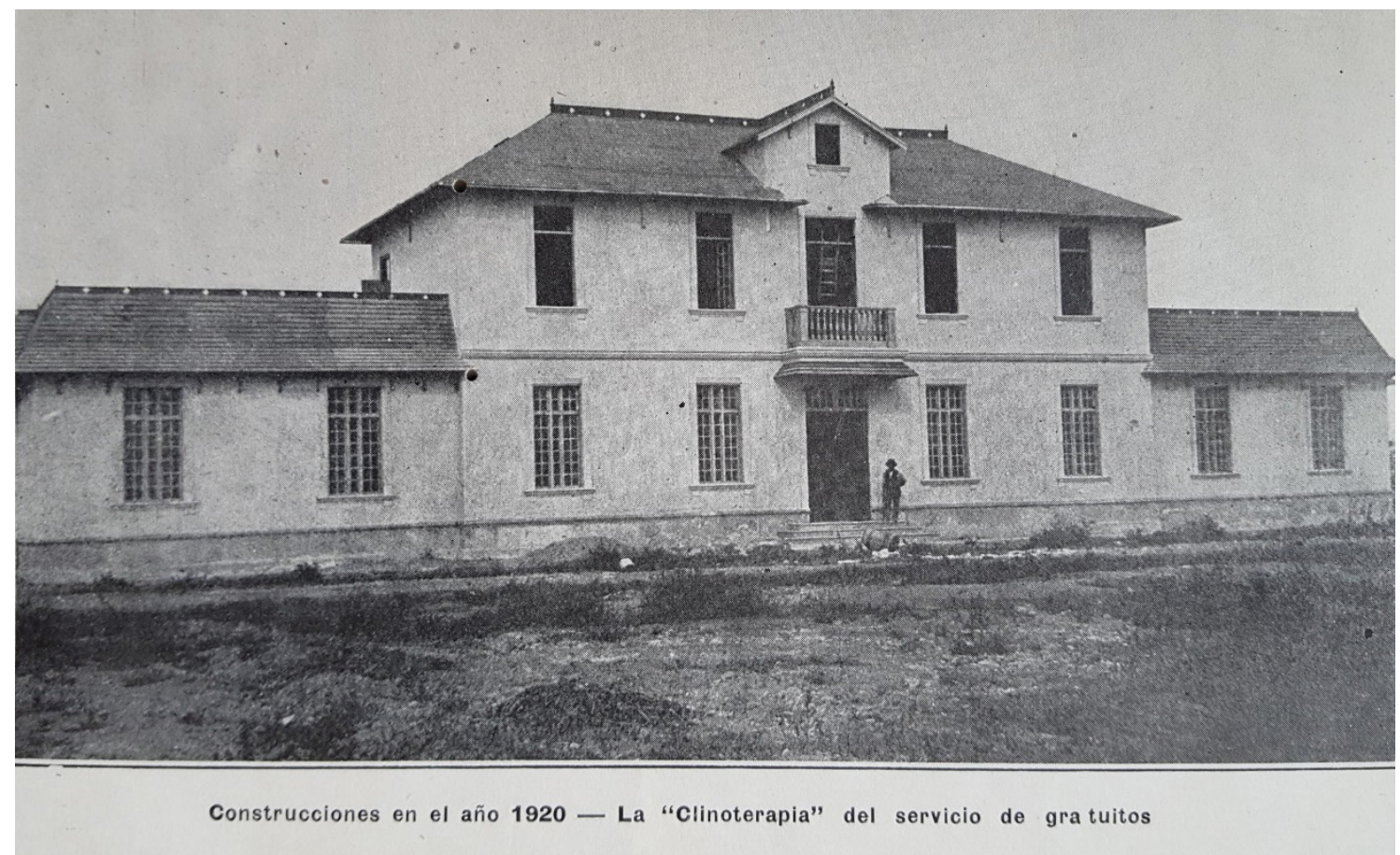

Figura 1. Uno de los pabellones de clinoterapia del Asilo Colonia de la Magdalena (14)

Regional Mixto de Alienados de Oliva, en Argentina, la laborterapia o "terapia activa" llevó al cierre del servicio de clinoterapia en 1929 (2).

Cabe agregar que la aparición y difusión de otros métodos terapéuticos como la malarioterapia, la insulinoterapia, el electrochoque y lapsicofarmacología fueron también desplazando a la clinoterapia en su sentido original. De este modo, alrededor de la década de 1950, Honorio Delgado consideraba que el tratamiento psiquiátrico era "casi nulo hasta comienzos del siglo", y se reducía "al empleo de sedantes, baños calientes prolongados y reposo en cama (clinoterapia)" (17). A finales de la década de 1950, en el Hospital Víctor Larco Herrera se denominaba "clino" al ambiente en donde se administraba la cura del sueño (información proporcionada por el Dr. Manuel Ponce Cornejo).

\section{Fisiología e indicaciones}

En líneas generales, se proponía que el sujeto sometido al reposo en el lecho mantenía la inactividad física; por tanto, los nervios sensitivos no producían "conciencia de movimiento" ni había excitación periférica, y como consecuencia había un "reposo psíquico", y se "eliminaba" así la causa de las emociones. Además, se describían cambios fisiológicos: disminución de la frecuencia cardíaca y respiratoria, descenso de la temperatura central, elevación de la presión arterial y disminución del peso (3).

Chaslin añadió otra explicación, relacionada a los preceptos del tratamiento moral, la cual postulaba que el alienado podía poseer una vaga consciencia de su enfermedad, o incluso tener intervalos de lucidez al estar en el lecho, con lo que comprendería de cierta manera que estaba "verdaderamente enfermo" y que necesitaba ser cuidado, lo que disipaba la desorientación (3).

La clinoterapia ocupaba un lugar de privilegio como tratamiento sintomático de diversas formas de locura, y se le reconocían efectos en conseguir una menor duración e intensidad en las psicosis. Notó Brandam que "desde la introducción de la clinoterapia en los asilos, son raros los cuadros clínicos con todo el complejo sintomático del delirio agudo y más raro aún verlos terminar por la muerte como ocurría en tiempos en que estos enfermos eran confinados en una estrecha celda" (3).

Su aplicación estaba dada tanto en formas agudas de locura como en episodios agudos de formas crónicas. Teniendo en cuenta la nosología de su época, Brandam mencionó la siguientes indicaciones (3):

- Psicosis tóxicas, como las causadas por alcohol, 
por enfermedades infecciosas (fiebre tifoidea, viruela) y por enfermedades metabólicas (pelagra).

- Locuras vesánicas.

- Melancolía, en la cual el reposo en el lecho se asumía como muy relevante, pues la actividad cerebral representaba una "tortura". A la par de la mejoría se iba relajando el reposo absoluto.

- Excitación maniaca, en la que, pese a la gran agitación inicial, se conseguía luego que el paciente permaneciera acostado.

- Psicosis de la pubertad (hebefrenia) y amentia de Meynert.

- Delirio sistematizado progresivo, si hubiera una exacerbación de su estado mental.

- Locura histérica.

- Locura epiléptica, si hubiera síntomas como excitación o impulsividad.

- Parálisis general progresiva.

- Locuras morfológicas, para facilitar su cuidado y cuando presentaban períodos de agitación.

- Cabe agregar aquí el uso ya mencionado de Mitchell en otros estados neuróticos tales como la neurastenia (8).

En cuanto a las enfermedades somáticas, como se mencionó previamente, el uso del reposo en el lecho acompaña a la historia de la medicina desde sus orígenes. En cuanto a menciones específicas del uso de la clinoterapia, referimos:

- Encefalopatias hipertensivas y arterioesclerosas (18).

- Enfermedad de Bouillaud (fiebre reumática), en la que se indicaba un reposo completo y prolongado en cama, con resultados prometedores: "sin duda alguna, la clinoterapia es el tratamiento de mayor eficacia para prevenir o disminuir las localizaciones cardiacas (...)" (19).

\section{Clinoterapia y medidas coercitivas}

El ruso Alexander Nikolaevich Bernstein resumió en los siguientes términos la expectativa generada por un manejo más humanitario del alienado con la práctica de la clinoterapia: "El régimen por el lecho es la última palabra del combate secular por la libertad y los derechos humanos del alienado" (3).

Era común ver en los asilos de la época enfermos recluidos en celdas, y aquí el reposo en el lecho fue el medio para sacarlos y colocarlos en grandes salas, donde también podrían ser observados con más cautela. "El nuevo régimen crea una nueva era en psiquiatría, permitiendo la observación clínica continuada y el estudio de las psicosis en conjunto, (...) este modo de tratamiento, hace olvidar la camisola de fuerza (...). El lecho hace desaparecer las celdas, (...) disminuye la intensidad de la agitación: no se ve más, esos estados congestivos que terminan, a menudo en el delirio agudo y la muerte; el sueño cuando sobreviene, es más reparador en el lecho, que en el piso de una celda; el enfermo es mejor cuidado, conoce mejor sus enfermeros y recíprocamente" (3).

No obstante, la mención a formas de tratamiento más restrictivo no eran raras: "En los casos de agitación considerable y ruidosa, el maníaco no es dejado en los dormitorios, por la noche: se le coloca en una cámara, cuya puerta provista de un ancho postigo, permite explorar y mirar, cuidando todas las partes de la pieza. Muchos de estos enfermos se someten voluntariamente al régimen: otros se resisten tenazmente, cediendo a la excitación motriz de que están animados. Estos últimos no permanecen tranquilos en el lecho y es necesario recurrir al aislamiento en cámara donde se encuentran mejor" (3).

Otra mención al uso concomitante de la clinoterapia con medidas más restrictivas (sujeción mecánica o camisa de fuerza) se encontró en un artículo publicado en Brasil. En este se menciona que el reposo sería la primera medida terapéutica a ser tomada, pero en el caso de pacientes más agitados, se podría "reforzar" la clinoterapia con el uso de amarras: la camisa de fuerza, las correas de contención y las sábanas, hasta hacer una especie de "envoltorio húmedo", que mantenía al paciente enrollado en sábanas mojadas por algunas horas. "Hubo algunos que rompieron las paredes por lo fuerte que las golpeaban, así que tuve que usar camisa de fuerza con puño (...) se amarraba al paciente con las manos hacia atrás, pues los pacientes de aquella época eran fuertes y agresivos (...) la contención mecánica era con una faja, como si se vendase a una persona (...) entonces el individuo estaba completamente inmóvil, no podía moverse durante ocho horas" (20).

Con el paso del tiempo, la clinoterapia terminó reducida en lo conceptual al simple aislamiento en una habitación estrecha, con el supuesto propósito de evitar la autolesión. Así, en el Perú, el Instituto Nacional de Salud Mental "Honorio Delgado - Hideyo Noguchi" contó desde su fundación, en 1982, con múltiples "ambientes de aislamiento" (21), que fueron más conocidos como "clinoterapia". Por su parte, la Defensoría del Pueblo, en el informe que elaboró luego de la supervisión a ocho establecimientos 
psiquiátricos del Perú en el 2004 (y publicado al año siguiente), señaló que "el aislamiento o clinoterapia se produce cuando la persona es conducida, con cuidado de no generar un daño en su persona, a un ambiente especialmente acondicionado para que pueda permanecer sola sin que se autoagreda" (22).

Lamentablemente, el uso de la clinoterapia, devenida en simple aislamiento en nuestro país, no siempre ha sido el idóneo. En el informe mencionado, la Defensoría del Pueblo "pudo observar un uso de la clinoterapia que podría encubrir un trato discriminatorio" o "con criterios de conveniencia para el funcionamiento del servicio (por ejemplo, para permitir la preparación de la medicación o la entrega del turno sin distracción)" (22). También en el 2004, la Asociación Mental Disability Rights International y la Asociación Pro Derechos Humanos "documentaron un uso inadecuado de los cuartos de reclusión (...). La deshidratación es un peligro real para los individuos mantenidos en reclusión durante largos periodos de tiempo" (23).

Como colofón mencionaremos que en marzo del 2020 se promulgó en el Perú el Reglamento de la Ley $N^{\circ} 30947$, Ley de Salud Mental, que en su artículo 28 prohíbe explícitamente "el uso de cualquier medida restrictiva en todos los servicios de salud, así como el uso de cualquier medida que vulnere los derechos de los(as) usuarios(as) tales como (...) el uso de cuartos de aislamiento" (24). Esto ya había sido incluido en la legislación de otros países. Por poner un ejemplo, el artículo 14 de la Ley Nacional de Salud Mental $N^{\circ}$ 26.657 de la Argentina, del año 2013, dice: "No será admitida la utilización de salas de aislamiento" (25).

\section{Correspondencia}

\section{Marco Macavilca-Cruz}

Correo electrónico: marco.macavilca@upch.pe.

\section{REFERENCIAS BIBLIOGRÁFICAS}

1. Gispert-Sauch AM. Raíces griegas: tan lejanas y tan cercanas. Letras. 2004;75 (107-108):197-205.

2. Eraso Y. "A burden to the state": The reception of the German "active therapy" in an Argentinean "Colony-Asylum" in the 1920s and 1930s. En: Ernst W, Mueller T (ed). Transnational Psychiatries: Social and Cultural Histories of Psychiatry in Comparative Perspective, c. 1800-2000. Cambridge: Cambridge Scholars Publishing; 2010. pp. 51-79. (Citado el 6 de septiembre del 2020). Disponible en: https://books. google.com.pe/books/about/Transnational_
Psychiatries.html?id=yIQ2SgAACAAJ\&redir_esc $=y$

3. Brandam J. La clinoterapia en psiquiatría. Contribución á su estudio. Tesis de Grado. Buenos Aires, Argentina: Universidad Nacional de Buenos Aires; 1901. (Citado el 6 de septiembre del 2020). Disponible en: http://www.bibliomedicinadigital. fmed.uba.ar/medicina/TESISH/Tesis_00955 Clinoterapia_en_psiquiatria_Brandam_Javier_1901. pdf

4. Macias L, Velásquez M. Glosario de referencias léxicas y culturales en la obra de León de Greiff. Medellín: Fondo Editorial Universidad EAFIT; 2007. (Citado el 6 de septiembre del 2020). Disponible en: https://books.google.com.pe/books?id=ipPhNb5tKU $\mathrm{cC} \&$ printsec $=$ frontcover\&hl $=\mathrm{es} \# \mathrm{v}=$ onepage $\& \mathrm{q} \& \mathrm{f}=\mathrm{f}$ alse

5. Ackerknecht E. Breve historia de la psiquiatría. Valencia: Godella; 1993.

6. Caravedo B. Organización de la asistencia de los alienados. En: Horvitz I (ed). Actas de las Primera Reunión de las Jornadas Neuro-Psiquiátricas Panamericanas. Santiago: Prensas de la Universidad de Chile; 1938:533-579 (Citado el 6 de septiembre del 2020). Disponible en: http://www.biblioteca. larcoherrera.gob.pe/documentos/jornadas/ jornadas1937_temaoficial7.pdf

7. Howe A, Sethi F. Seclusion: the untold legacy of the non-restraintmovementintheUK.JPsychiatrIntensive Care. 2018;14(1):5-13. (Citado el 6 de septiembre del 2020). Disponible en: https://www.ingentaconnect. com/content/napicu/jpic/2018/00000014/00000001/ $\operatorname{art} 00002$

8. Gilman CP, Charlotte P, Gilman P. The rest cure revisited. Am J Psychiatry. 2007;164(5):737-8.

9. Ruggiero G. Eating disorders in the Mediterranean Area: An Exploration in Transcultural Psychology. New York: Nova Science Publishers; 2004. (Citado el 6 de septiembre del 2020). Disponible en: https:// books.google.com.pe/books?id=rwbZUmSrjdgC\&pr intsec $=$ frontcover $\& \mathrm{hl}=\mathrm{es} \# \mathrm{v}=$ onepage $\& \mathrm{q} \& \mathrm{f}=$ false

10. Rossi L, Jardon M. Historias de vida en las historias clínicas. Instituciones, prácticas y sujeto implicado. Anuario de Investigaciones. 2014;21:185-192.

11. Sitjes F. Ecos de la vida en el Hospicio. La visita ministerial. Buenos Aires: Polemos. 1998. (Citado el 6 de septiembre del 2020). Disponible en: http:// www.polemos.com.ar/docs/temas/Temas $4 / 2 \mathrm{a} \% 20$ p.\%2002\%20visita\%20miniterial.htm

12. Dellacasa C. "Ecos de las Mercedes": El órgano de la chifladura. Temas de historia de la Psiquiatría Argentina. Buenos Aires: Polemos; 1998. p. 4. (Citado el 6 de septiembre del 2020). Disponible en: http:// www.polemos.com.ar/docs/temas/Temas4/1.\%20 Primera\%20parte.htm

13. Venancio A. La asistencia psiquiátrica en la historia política brasileña. Asclepio. Revista de Historia de la 
Medicina y de la Ciencia. 2012; 64(1):167-188.

14. Sociedad de Beneficencia Pública de Lima. Memoria correspondiente al año de 1920. Lima: Casa Editora M. Moral; 1920.

15. Hospital "Víctor Larco Herrera". Memoria de la Dirección. Correspondiente al año de 1948. Lima: Talleres Gráficos del Hospital "Víctor Larco Herrera"; 1952.

16. Turnes AL. Historia y evolución de los hospitales en las diferentes culturas. Montevideo: Sindicato Médico del Uruguay; 2009. (Citado el 6 de septiembre del 2020). Disponible en: https://www.smu.org.uy/ $\mathrm{dpmc} / \mathrm{hmed} /$ historia/articulos/origen-y-evolucion.pdf

17. Delgado H. Florecimiento de la psiquiatría. Revista de Neuro-Psiquiatría. 1992; 55(3):151-155.

18. Rodriguez-Arias B. Terapéutica médica de las encefalopatías hipertensivas y arterioesclerosas. Ensayos con derivados piridoxínicos. Anales de Medicina y Cirugía. 1960;40(159):155-158.

19. Macera J, Ruchelli A, Gaig R. Profilaxis y tratamiento del reumatismo (Enfermedad de Bouillaud) en la infancia. Revista Chilena de Pediatría. 1942;13(23):278-290.

20. Lappann-Botti N. Uma viagem na história da enfermagem psiquiátrica no início do século XX. Esc Anna Nery R Enferm. 2007;10(4):725-729.

21. Mariátegui J. Salud mental y realidad nacional. El primer quinquenio del Instituto Nacional de Salud Mental. Lima: Asociación Psiquiátrica Peruana; 1988.
22. Defensoría del Pueblo. Salud mental y derechos humanos: La situación de los derechos de las personas internadas en establecimientos de salud mental. Informe Defensorial $\mathrm{N}^{\mathrm{o}}$ 102. Lima: Defensoría del Pueblo; 2005. (Citado el 6 de septiembre del 2020). Disponible en: https:// w w w.defensoria.gob.pe/w p - content/ uploads/2005/12/informe_102.pdf

23. Mental Disability Rights International y Asociación Pro Derechos Humanos. Derechos humanos \& salud mental en el Perú. Lima: Mental Disability Rights International y Asociación Pro Derechos Humanos; 2004. (Citado el 6 de septiembre del 2020). Disponible en: https://www.driadvocacy.org/wpcontent/uploads/Peru-Report-Esp-Final-Spanish.pdf

24. Ministerio de Salud. Decreto Supremo N 007-2020SA. Decreto Supremo que aprueba el Reglamento de la Ley $\mathrm{N}^{\circ}$ 30947, Ley de Salud Mental. Lima: Diario Oficial El Peruano; 5 de marzo del 2020. (Citado el 6 de septiembre del 2020). Disponible en: https://busquedas.elperuano.pe/download/url/ decreto-supremo-que-aprueba-el-reglamentode-la-ley-n-30947-decreto-supremo-n-007-2020sa-1861796-1

25. Ley Nacional de Salud Mental N ${ }^{\circ}$ 26.657. Decreto Reglamentario 603/2013Buenos Aires: Ministerio de Salud; 2013. (Citado el 6 de septiembre del 2020). Disponible en: http://www.msal.gob.ar/images/ stories/ryc/graficos/0000001237cnt-2018_leynacional-salud-mental-decreto-reglamentario.pdf

Recibido: 01/06/2019

Aceptado: 14/09/2020 